FACTA UNIVERSITATIS (NIŠ)

Ser. Math. Inform. Vol. 35, No 2 (2020), 523-531

https://doi.org/10.22190/FUMI2002523Y

\title{
HYPERBOLIC TYPE SOLUTIONS FOR THE COUPLE BOITI-LEON-PEMPINELLI SYSTEM
}

\author{
Asif Yokuş, Hülya Durur and Hijaz Ahmad*
}

(C) 2020 by University of Niš, Serbia | Creative Commons Licence: CC BY-NC-ND

\begin{abstract}
In this paper, the $\left(1 / G^{\prime}\right)$-expansion method is proposed to construct hyperbolic type solutions of the nonlinear evolution equations. To asses the applicability and effectiveness of the method, two cases of the coupled Boiti-Leon-Pempinelli (CBLP) system have been investigated in this study. It is shown that, with the help of symbolic computation, the $\left(1 / G^{\prime}\right)$-expansion method provides a powerful and straightforward mathematical tool for solving nonlinear partial differential equations.
\end{abstract}

Keywords: nonlinear evolution equations; partial differential equations; symbolic computation.

\section{Introduction}

Nonlinear evolution equations usually used to describe the nonlinear phenomena of waves in plasma physics, ocean engineering, quantum mechanics, fluid dynamics, solid state physics, hydrodynamics and many other branches of sciences and engineering. These types of equations have been used to describe the liquid flow containing gas bubbles, the propagation of waves, fluid flow in elastic oceans, rivers, tubes, lakes as well as a gravity waves in a smaller domain and Spatio-temporal rescaling of the nonlinear wave motion.

There are several approaches for finding solutions of nonlinear partial differential equations which have been developed and employed successfully. Some of these are a new sub equation method [1], homotopy analysis method [2,3], homotopy-Pade method [4], homotopy perturbation method [5, 6], $\left(G^{\prime} / G\right)$-expansion method [7, 8], modified variational iteration algorithm-I [9, 10, 11], sub equation method [12], Variational iteration method with an auxiliary parameter $[13,14,15,16]$, sumudu transform approach [17], $\left(1 / G^{\prime}\right)$-expansion method [18, 19], variational iteration method [20, 21], auto-Bäcklund transformation method [22], Clarkson-Kruskal direct method [23], Bernoulli sub-equation function technique [24], decomposition

Received April 16, 2020; accepted May 03, 2020

2010 Mathematics Subject Classification. Primary 39A14; Secondary 35C07

${ }^{*}$ Corresponding author Email: hijaz555@gmail.com 
method [25, 26, 27, 28], modified variational iteration algorithm-II [29, 30, 31], first integral method [32], homogeneous balance method [33], modified Kudryashov technique [34], residual power series approach [35], collocation method [36], extended rational SGEEM [37], sine-Gordon expansion method [38, 39] and many more $[40,41,42,43]$.

Consider the following coupled Boiti-Leon-Pempinelli System [44]

$$
\begin{aligned}
& u_{t y}=\left(u^{2}-u_{x}\right)_{x y}+2 v_{x x x}, \\
& v_{t}=v_{x x}+2 u v_{x} .
\end{aligned}
$$

There have been numerous studies about the analytical treatment of CBLP System. In some of the studies, new traveling wave solutions of CBLP System have been attained utilizing the generalized $\left(G^{\prime} / G\right)$-expansion method [44], while the analytic solutions of CBLP System have been obtained in [45].

In current work, we will construct the exact solutions of the CBLP System employing $\left(1 / G^{\prime}\right)$-expansion method.

The remaining portion of this paper is as follows: In section $2,\left(1 / G^{\prime}\right)$-expansion method is elaborated, in section $3,\left(1 / G^{\prime}\right)$-expansion method's applications are discussed and utilized to obtain hyperbolic type solutions of the CBLP System, applicability and reliability of the proposed techniques are shown through 3D, contour and 2D graphics. The conclusion is discussed in the last section.

\section{Description of the Method}

Consider a general form of the following nonlinear PDE,

$$
\sigma\left(u, \frac{\partial u}{\partial t}, \frac{\partial u}{\partial x}, \frac{\partial u}{\partial y}, \frac{\partial^{2} u}{\partial x^{2}}, \ldots\right)=0 .
$$

Here, let $u=u(\xi)=u(x, y, t), \quad \xi=x+y-c t, \quad c \neq 0$, where $c$ is a constant and the speed of the wave. We can convert it into the following nODE for $u(\xi)$

$$
\tau\left(u,-c u^{\prime}, u^{\prime}, u^{\prime}, u^{\prime \prime}, \ldots\right)=0 .
$$

The solution of Eq. (2.2) is assumed to have the form

$$
u(\xi)=a_{0}+\sum_{i=1}^{n} a_{i}\left(\frac{1}{G^{\prime}}\right)^{i},
$$

whereas $a_{i}, \quad i=0,1, \ldots, n$ are nonzero constants, $G=G(\xi)$ provides the following second order IODE

$$
G^{\prime \prime}+\lambda G^{\prime}+\mu=0,
$$

where $\mu$ and $\lambda$ are constants to be determined after,

$$
\frac{1}{G^{\prime}(\xi)}=\frac{1}{-\frac{\mu}{\lambda}+B \cosh [\xi \lambda]-B \sinh [\xi \lambda]},
$$


where $B$ is integral constant. If the desired derivatives of the Eq. (2.3) are calculated and substituting in the Eq. (2.2), a polynomial with the argument $\left(1 / G^{\prime}\right)$ is attained. An algebraic equation system is created by equalizing the coefficients of this polynomial to zero. The equation are solved using a package program and put into place in the default Eq. (2.2) solution function. Lastly, the solutions of Eq. (1.1) are found.

\section{Solutions of CBLP System}

The traveling wave transmutation $\xi=x+y-c t$, allows us to convert Eq. (1.1) into an ODE for $u=u(\xi)$

$$
\begin{gathered}
-c u^{\prime \prime}=\left(u^{2}-u^{\prime}\right)^{\prime \prime}+2 v, \\
-c v^{\prime}=v^{\prime \prime}+2 u v^{\prime},
\end{gathered}
$$

here by integrating twice the Eq. (3.1), we attain

$$
v^{\prime}=\frac{1}{2} u^{\prime}-\frac{1}{2} c u-\frac{1}{2} u^{2}
$$

According to $\xi$ in Eq. (3.3) and considering zero constants for integration, we attain

$$
v=\frac{1}{2} u-\frac{1}{2} \int\left(c u+u^{2}\right) d \xi .
$$

Replacing Eq. (3.3) into the Eq. (3.2),

$$
u^{\prime \prime}-2 u^{3}-3 c u^{2}-c^{2} u=0 .
$$

In Eq. (3.5), we get balancing term $n=1$ and in Eq.(2.3), the following situation is obtained:

$$
u(\xi)=a_{0}+a_{1}\left(\frac{1}{G^{\prime}}\right), \quad a_{1} \neq 0 .
$$

Replacing Eq. (3.6) into Eq. (3.5) and the coefficients of the algebraic Eq. (1.1) are equal to zero, can find the following algebraic equation systems

$$
\begin{aligned}
& \text { Const }:-c^{2} a_{0}-3 c a_{0}^{2}-2 a_{0}^{3}=0, \\
& \left(\frac{1}{G^{\prime}[\xi]}\right)^{1}:-c^{2} a_{1}+\lambda^{2} a_{1}-6 c a_{0} a_{1}-6 a_{0}^{2} a_{1}=0, \\
& \left(\frac{1}{G^{\prime}[\xi]}\right)^{2}: 3 \lambda \mu a_{1}-3 c a_{1}^{2}-6 a_{0} a_{1}^{2}=0, \\
& \left(\frac{1}{G^{\prime}[\xi]}\right)^{3}: 2 \mu^{2} a_{1}-2 a_{1}^{3}=0 .
\end{aligned}
$$

\section{Case1:}

$$
a_{0}=0, a_{1}=-\mu, c=-\lambda,
$$

replacing Eq.(3.8) into the Eq.(3.6) and the following hyperbolic type solutions is obtained for Eq. (1.1): 


$$
u_{1}(x, y, t)=-\frac{\mu}{-\frac{\mu}{\lambda}+B \cosh [\lambda(t \lambda+x+y)]-B \sinh [\lambda(t \lambda+x+y)]}
$$$$
v_{1}(x, y, t)=\frac{1}{4 \mu}\left(\lambda \mu\left(\lambda(t \lambda+x+y)+2 \log \left[\begin{array}{c}
(-B \lambda+\mu) \cosh \left[\frac{1}{2} \lambda(t \lambda+x+y)\right] \\
+(B \lambda+\mu) \sinh \left[\frac{1}{2} \lambda(t \lambda+x+y)\right]
\end{array}\right]\right)\right.
$$$$
-\lambda \mu\left(\lambda(t \lambda+x+y)+2 \log \left[\begin{array}{l}
(-B \lambda+\mu) \cosh \left[\frac{1}{2} \lambda(t \lambda+x+y)\right] \\
+(B \lambda+\mu) \sinh \left[\frac{1}{2} \lambda(t \lambda+x+y)\right]
\end{array}\right]\right.
$$

$$
-\frac{4 B \lambda \mu \sinh \left[\frac{1}{2} \lambda(t \lambda+x+y)\right]}{\left.(A \lambda-\mu)\left(\begin{array}{c}
(-B \lambda+\mu) \cosh \left[\frac{1}{2} \lambda(t \lambda+x+y)\right] \\
+(B \lambda+\mu) \sinh \left[\frac{1}{2} \lambda(t \lambda+x+y)\right]
\end{array}\right)\right)}
$$

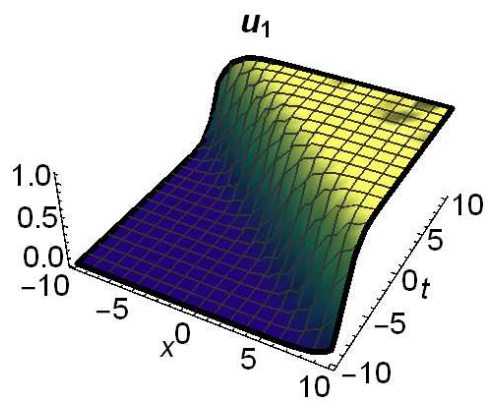

$v_{1}$

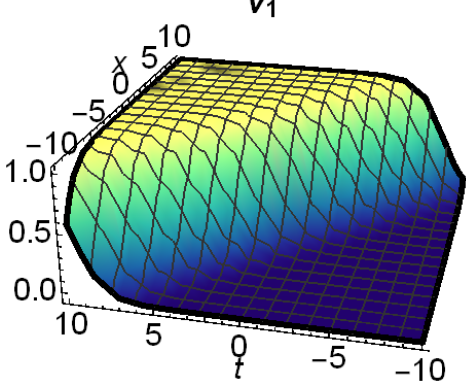

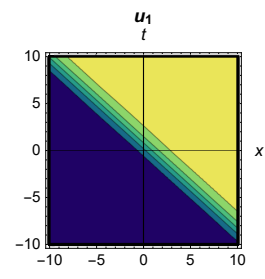
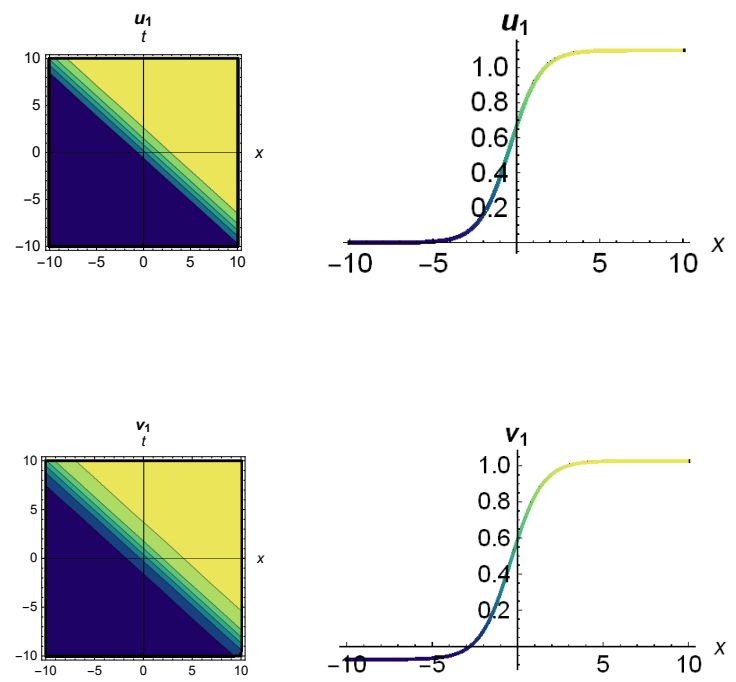

FIG. 3.1: 3D, contour and 2D graphs respectively for $B=0.6, \mu=-0.1, y=$ $1, \lambda=1.1$ values of Eqs. (3.9) and (3.10).

\section{Case 2:}

$$
a_{0}=-\lambda, a_{1}=-\mu, c=\lambda,
$$

replacing values Eq. (3.11) into Eq. (3.6) and the following hyperbolic type solutions are obtained for Eq. (1.1):

$\left(3.12 \psi_{2}(x, y, t)=-\lambda-\frac{\mu}{-\frac{\mu}{\lambda}+B \cosh [\lambda(x-t \lambda+y)]-B \sinh [\lambda(x-t \lambda+y)]}\right.$, 


$$
\begin{aligned}
& v_{2}(x, y, t)=\frac{1}{2}\left(\lambda^{2}(-x-y+t \lambda)\right. \\
& +\frac{1}{\mu} \lambda\left(\lambda(x-t \lambda+y) \mu+\mu\left(\lambda(x-t \lambda+y)+2 \log \left[\begin{array}{c}
\left.\left.\left.(-B \lambda+\mu) \cosh \left[\frac{1}{2} \lambda(x+y-t \lambda)\right]+\right]\right)\right) \\
(B \lambda+\mu) \sinh \left[\frac{1}{2} \lambda(x+y-t \lambda)\right]
\end{array}\right]\right)\right. \\
& +\frac{1}{2 \mu}\left(-\lambda \mu\left(\lambda(x-t \lambda+y)+2 \log \left[\begin{array}{c}
(-B \lambda+\mu) \cosh \left[\frac{1}{2} \lambda(x-t \lambda+y)\right] \\
+(B \lambda+\mu) \sinh \left[\frac{1}{2} \lambda(x-t \lambda+y)\right]
\end{array}\right]\right)\right. \\
& -\lambda \mu\left(\lambda(x-t \lambda+y)+2 \log \left[\begin{array}{c}
(-B \lambda+\mu) \cosh \left[\frac{1}{2} \lambda(x-t \lambda+y)\right] \\
+(B \lambda+\mu) \sinh \left[\frac{1}{2} \lambda(x-t \lambda+y)\right]
\end{array}\right]\right) \\
& -\frac{4 B \lambda \mu \sinh \left[\frac{1}{2} \lambda(x-t \lambda+y)\right]}{\left.(B \lambda-\mu)\left(\begin{array}{c}
(-B \lambda+\mu) \cosh \left[\frac{1}{2} \lambda(x-t \lambda+y)\right] \\
+(B \lambda+\mu) \sinh \left[\frac{1}{2} \lambda(x-t \lambda+y)\right]
\end{array}\right)\right)} \\
& +\frac{1}{2}\left(-\lambda-\frac{\mu}{-\frac{\mu}{\lambda}+B \cosh [\lambda(x-t \lambda+y)]-B \sinh [\lambda(x-t \lambda+y)]}\right) .
\end{aligned}
$$
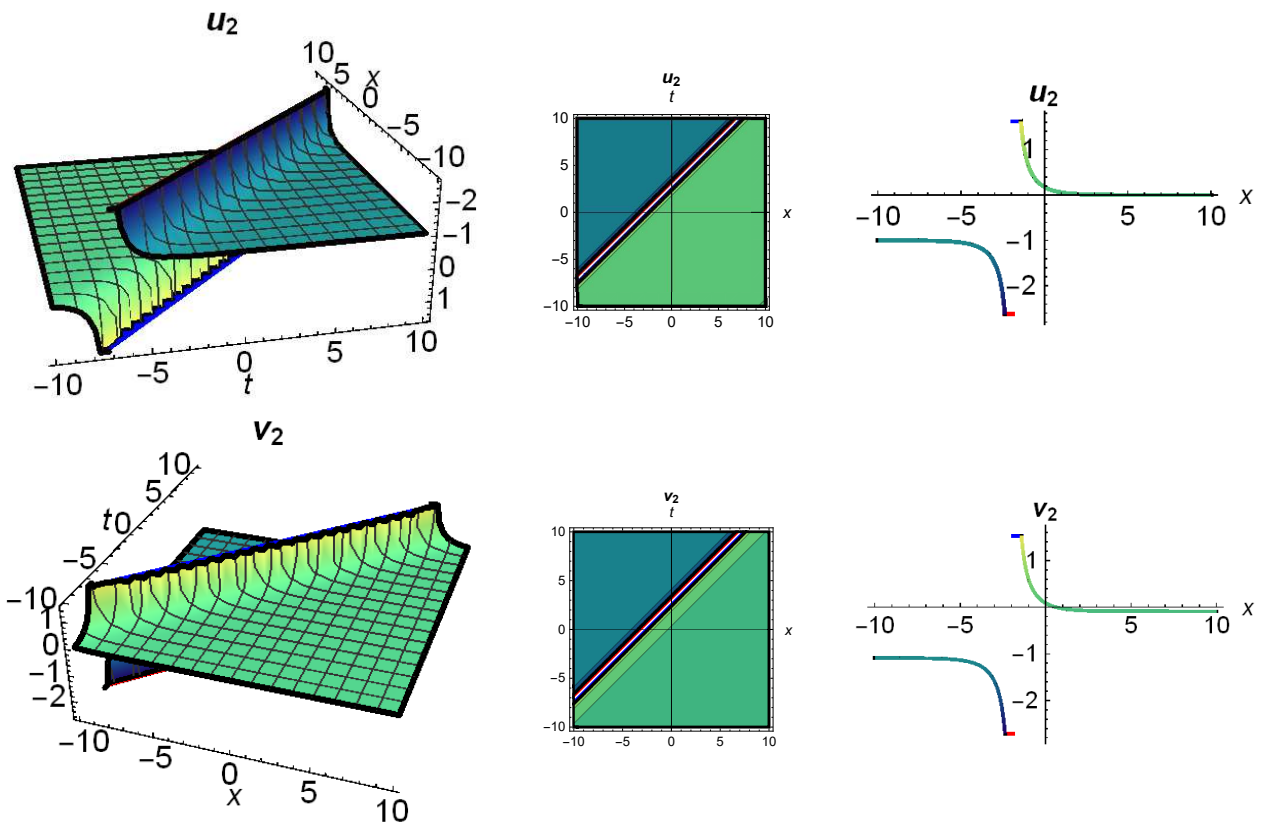

FIG. 3.2: 3D, contour and 2D graphs respectively for $B=0.6, \mu=-0.1, y=$ $1, \lambda=1.1$ values of Eqs. (3.12) and (3.13).

\section{Conclusion}

In this work, we have achieved hyperbolic type exact solutions of the CBLP System with the help of $\left(1 / G^{\prime}\right)$-expansion method. Computer technology utilized in the construction of $3 \mathrm{D}, 2 \mathrm{D}$ and contour graphics of the obtained solutions. The CBLP System, which plays an important role in mathematical physics, has been investigated analytically for the effectiveness and reliability of the proposed method. 
Furthermore, the applied method is an effective, powerful method and can be used to establish new exact solutions of many other nonlinear partial differential equations arising in applied sciences and engineering.

\section{R E F E R E N C E S}

1. A. Kurt, O. TaşBozan and H. Durur: The Exact Solutions of Conformable Fractional Partial Differential Equations Using New Sub Equation Method. Fundamental Journal of Mathematics and Applications, 2019, 2(2), pp. 173-179.

2. H. M. Sedighi And K. H. ShirazI: Using homotopy analysis method to determine profile for disk cam by means of optimization of dissipated energy. International Review of Mechanical Engineering, 2011, 5(5), pp. 941-946.

3. J. H. HE: Comparison of homotopy perturbation method and homotopy analysis method. Applied Mathematics and Computation, 2004, 156(2), pp. 527-539.

4. H. Kheiri, N. Alipour and R. Dehghani: Homotopy analysis and Homotopy-Pade methods for the modified Burgers-Korteweg-de-Vries and the Newell Whitehead equation. Mathematical Sciences, 2011, 5(1), pp. 33-50.

5. H. M. Sedighi And F. Daneshmand: Nonlinear transversely vibrating beams by the homotopy perturbation method with an auxiliary term. Journal of Applied and Computational Mechanics, 2014, 1(1), pp. 1-9.

6. J. H. HE: Homotopy perturbation method: a new nonlinear analytical technique. Applied Mathematics and computation, 2003, 135(1), pp. 73-79.

7. A. YoKuş AND D. KAYA: Traveling wave solutions of some nonlinear partial differential equations by using extended-expansion method. Advances in Mathematical Physics, 2015.

8. H. DuRuR: Different types analytic solutions of the $(1+1)$-dimensional resonant nonlinear Schrödinger's equation using $\left(G^{\prime} / G\right)$-expansion method. Modern Physics Letters B, 2020, 34(03).

9. H. Ahmad, T. A. Khan and C. Cesarano: Numerical Solutions of Coupled Burgers' Equations. Axioms, 2019, 8(4).

10. H. Ahmad, T. A. Khan And S. YaO: Numerical solution of second order Painlevé differential equation. Journal of Mathematics and Computer Science, 2020, 21(2), pp. 150-157.

11. H. Ahmad, A.R. Seadawy, T.A. Khan and P. Thounthong: Analytic approximate solutions for some nonlinear Parabolic dynamical wave equations. Journal of Taibah University for Science, 2020, 14(1), pp. 346-358.

12. H. Durur, O. Taşbozan, A. Kurt and M. Şenol: New Wave Solutions of Time Fractional Kadomtsev-Petviashvili Equation Arising In the Evolution of Nonlinear Long Waves of Small Amplitude. Erzincan University Journal of the Institute of Science and Technology, 2019, 12(2), pp. 807-815.

13. H. AhmaD: Variational iteration method with an auxiliary parameter for solving differential equations of the fifth order. Nonlinear Science Letters A. 2018, 9(1), pp. 27-35.

14. M. Rafiq, H. Ahmad, S.T. Mohyud-Din: Variational iteration method with an auxiliary parameter for solving Volterra's population model. Nonlinear Science Letters A. 2017, 8(4), pp. 389-396. 
15. H. Ahmad, M. Rafiq, C. Cesarano And H. Durur: Variational iteration algorithm-I with an auxiliary parameter for solving boundary value problems. Earthline Journal of Mathematical Sciences, 2020, 3(2), pp. 229-247.

16. H. AHMAD AND T. A. KHAN: Variational iteration algorithm-I with an auxiliary parameter for wave-like vibration equations. Journal of Low Frequency Noise, Vibration and Active Control, 2019, 38(3-4), pp. 1113-1124.

17. M. YAVUZ AND N. ÖzDEmiR: An Integral Transform Solution for Fractional Advection-Diffusion Problem. Mathematical Studies and Applications, 2018, 4-6 October, 442 .

18. A. Yokuş AND H. DuRuR: Complex hyperbolic traveling wave solutions of KuramotoSivashinsky equation using $\left(1 / G^{\prime}\right)$ expansion method for nonlinear dynamic theory. Journal of Balıkesir University Institute of Science and Technology, 2019, 21(2), pp. 590-599.

19. H. Durur And A. YoKuş: $\left(1 / G^{\prime}\right)$-Açılım Metodunu Kullanarak Sawada-Kotera Denkleminin Hiperbolik Yürüyen Dalga Çözümleri. Afyon Kocatepe Üniversitesi Fen ve Mühendislik Bilimleri Dergisi, 2019, 19(3), pp. 615-619.

20. J. H. HE: Variational iteration method for autonomous ordinary differential systems. Applied Mathematics and Computation, 2000, 114(2-3), pp. 115-123.

21. J. H. HE: Variational iteration method-some recent results and new interpretations. Journal of computational and applied mathematics, 2007, 207(1), pp. 3-17.

22. D. Kaya, A. Yokuş And U. DemiroĞLu: Comparison of Exact and Numerical Solutions for the Sharma-Tasso-Olver Equation. In Numerical Solutions of Realistic Nonlinear Phenomena, 2020, pp. 53-65. Springer, Cham.

23. Q. Su-Ping And T. Li-XIN: Modification of the Clarkson-Kruskal Direct Method for a Coupled System. Chinese Physics Letters, 2007, 24(10), 2720.

24. F. Dusunceli: New Exact Solutions for Generalized $(3+1)$ Shallow Water-Like (SWL) Equation. Applied Mathematics and Nonlinear Sciences, 2019, 4(2), pp. 365370.

25. D. KAYA AND A. YoKUŞ: A numerical comparison of partial solutions in the decomposition method for linear and nonlinear partial differential equations. Mathematics and Computers in Simulation, 2002, 60(6), pp. 507-512.

26. D. KAYA AND A. YoKUŞ: A decomposition method for finding solitary and periodic solutions for a coupled higher-dimensional Burgers equations. Applied Mathematics and Computation, 2005, 164(3), pp. 857-864.

27. A. YoKUS AND D. KAYA: A numerical comparison for coupled boussinesq equations by using the ADM. In: Proceedings, 2004, pp. 730-736.

28. M. YAVUZ AND N. ÖZDEMIR: A quantitative approach to fractional option pricing problems with decomposition series. Konuralp Journal of Mathematics, 2018, 6(1), pp. $102-109$.

29. H. Ahmad, T.A. Khan, P. S. Stanimirovic and I. Ahmad: Modified Variational Iteration Technique for the Numerical Solution of Fifth Order KdV Type Equations. Journal of Applied and Computational Mechanics, 2020.

30. H. Ahmad, A. R. Seadawy, T. A. Khan: Study on numerical solution of dispersive water wave phenomena by using a reliable modification of variational iteration algorithm. Mathematics and Computers in Simulation, 2020. 
31. H. Ahmad, A. R. Seadawy, T. A. Khan: Numerical solution of Korteweg-de VriesBurgers equation by the modified variational iteration algorithm-II arising in shallow water waves. Physica Scripta, 2020, 95(4).

32. M. Darvishi, S. Arbabi, M. NAJAfi And A. Wazwaz: Traveling wave solutions of a (2+1)-dimensional Zakharov-like equation by the first integral method and the tanh method. Optik, 2016, 127(16), pp. 6312-6321.

33. A. A. Rady, E. S. Osman And M. Khalfallah: The homogeneous balance method and its application to the Benjamin-Bona-Mahoney (BBM) equation. Applied Mathematics and Computation, 2010, 217(4), pp. 1385-1390.

34. D. Kumar, A. R. Seadawy and A. K. JoArdar: Modified Kudryashov method via new exact solutions for some conformable fractional differential equations arising in mathematical biology. Chinese journal of physics, 2018, 56(1), pp. 75-85.

35. H. Durur, M. Şenol, A. Kurt And O. TaşBozan: Zaman-Kesirli KadomtsevPetviashvili Denkleminin Conformable Türev ile Yaklaşık Çözümleri. Erzincan University Journal of the Institute of Science and Technology, 2019, 12(2), pp. 796-806.

36. I. AzIZ AND B. ŠARLER: The numerical solution of second-order boundary-value problems by collocation method with the Haar wavelets. Mathematical and Computer Modelling, 2010, 52(9-10), pp. 1577-1590.

37. T. A. Sulaiman And H. Bulut: The new extended rational SGEEM for construction of optical solitons to the (2+1)-dimensional Kundu-Mukherjee-Naskar model. Applied Mathematics and Nonlinear Sciences, 2019, 4(2), pp. 513-522.

38. H. M. Baskonus, H. Bulut And T. A. Sulaiman: New complex hyperbolic structures to the lonngren-wave equation by using sine-gordon expansion method. Applied Mathematics and Nonlinear Sciences, 2019, 4(1), pp. 129-138.

39. E. İ. EskitaşÇıoĞLu, M. B. Aktaş And H. M. Baskonus: New complex and hyperbolic forms for Ablowitz-Kaup-Newell-Segur wave equation with fourth order. Applied Mathematics and Nonlinear Sciences, 2019, 4(1), pp. 105-112.

40. A. YokUs AND S. GÜlBAhAR: Numerical solutions with linearization techniques of the fractional harry dym equation. Applied Mathematics and Nonlinear Sciences, 2019, 4(1), pp. 35-42.

41. H. AhmAD: Variational iteration algorithm-II with an auxiliary parameter and its optimal determination. Nonlinear Science Letters A, 2018, 9(1), pp. 62-72.

42. H. M. Sedighi, K. H. Shirazi And M. A. Attarzadeh: A study on the quintic nonlinear beam vibrations using asymptotic approximate approaches. Acta Astronautica, 2013, 19, pp. 245-250.

43. H. Ahmad, T. A. Khan: Variational iteration algorithm I with an auxiliary parameter for the solution of differential equations of motion for simple and damped mass-spring systems. Noise \& Vibration Worldwide, 2020, 51(1-2), pp. 12-20.

44. M. F. Aghdaei And J. M. Heris: Exact Solutions of the Couple Boiti-LeonPempinelli System by the Generalized $\left(G^{\prime} / G\right)$-expansion Method. Journal of Mathematical Extension, 2011, 5(2), pp. 91-104.

45. M. A. Abdelrahman And M. M. Khater: Traveling wave solutions for the couple Boiti-Leon-Pempinelli system by using extended Jacobian elliptic function expansion method. Journal of Advances in Physics, 2019, 11(3). 
Asif Yokuş

Faculty of Science

Department of Actuary

Firat University

Elazig, 23100, Turkey

asfyokus@yahoo.com

Hülya Durur

Faculty of Engineering

Department of Computer Engineering

Ardahan University

Ardahan, 75000, Turkey

hulyadurur@ardahan.edu.tr

Hijaz Ahmad

Department of Basic Sciences

University of Engineering and Technology

Peshawar 25000, Pakistan

hijaz555@gmail.com 